\title{
Breast Juvenile Fibroadenoma
}

National Cancer Institute

\section{Source}

National Cancer Institute. Breast /uvenile Fibroadenoma. NCI Thesaurus. Code C4276.

A breast fibroadenoma characterized by epithelial hyperplasia and an increased stromal cellularity. 